\title{
Improved Path Planning of Mobile Robot using Atom Search Optimization Algorithm
}

\author{
Shahad M.Majeed ${ }^{1}$, Issa Ahmed Abed ${ }^{2}$, and Alaa A. Al-Saffar ${ }^{3}$ \\ shahadmohammed@stu.edu.iq ${ }^{1}$, issaahmedabed@stu.edu.iq ${ }^{2}$, alaaasaffar@stu.edu.iq ${ }^{3}$ \\ Basrah Engineering Technical College, Southern Technical University, Iraq
}

\begin{abstract}
Robots have found applications in a variety of settings, ranging from speeding up manufacturing processes for completing complex jobs in hazardous and hostile areas. Path planning for mobile robots is one of the most difficult challenges in robotics. Robot route planning is a difficult optimization issue that must be tackled in a variety of applications. In this paper, we proposed the Atom search optimization (ASO) algorithm to improve the mobile robot path with free, static and dynamic obstacles environments and compare the results with the particle swarm optimization (PSO) algorithm. The "atom search optimization" algorithm is a novel algorithm that was used for the first time in this study to plan the route of a mobile robot. This algorithm has improved the path and the execution time(time required for the robot to reach the destination) was reduced. The authors checked the proposed method with several cases using MATLAB2020a software. It has been demonstrated that the suggested strategy, rather than PSO, found better, more consistent, and smoother pathways with a shorter computation time.
\end{abstract}

Keywords. Mobile robot route planning, Atom search optimization algorithm, particle swarm optimization.

\section{Introduction}

Rapid technological advancements open up new and better prospects in a variety of areas. Robotics is now one of the most exciting developments [1]. The primary aim of robotic technology is to develop machines that can perform such human tasks. They are commonly used in a variety of circumstances to speed up a process or work in and exploring aggressive, hazardous, and inaccessible environments [1]. Forward kinematics and, later, inverse kinematics is necessary for the design of any work in robotics systems [2]. In this work Forward kinematics is considered. One issue is how the mobile robot determines the shortest path to the target while avoiding collisions. In mobile robot work, the path planning issue is crucial. To plan the course of the robot from one point to another, robotic systems employ intelligence algorithms. Path planning's main objective is to determine a robot's permissible movement in a given setting with obstacles. From the start position to the target position, these motions require a collision-free direction [2]. A smart mobile robot route planning system can save a lot of time while also lowering the robot's wear and capital expenditure 
[3]. Researchers all around the world have developed a variety of meta-heuristic search algorithms to address complicated global optimization issues and approaches to improve them during the last few decades [5]. A constrained optimization problem can be used to overcome the difficulty of route planning. Depending on the environment and tasks that the robot must do, the goal or fitness function of the problem can be presented in a variety of ways [1].

In this paper, For tackling mobile robot path planning in two-dimensional space with static and dynamic impediments, the (ASO) technique was introduced. The results are compared with a PSO algorithm in the same manner. The suggested technique is a novel type of atom dynamics-based meta-heuristic global optimization methodology. ASO is a population-based iterative heuristic global optimization algorithm that that can be applied to a wide range of optimization issues. ASO is a simple and straightforward method that mathematically describes and replicates nature's atomic motion concept, where atom interaction forces emerging from the Lennard-Jones potential and constraint forces arising from the bond-length potential interact with each other. To investigate the problem of mobile robot path planning, the ASO method is utilized. In path planning issues, The findings of the experiments indicate that ASO can outperform other well-known techniques such as (PSO) and that it is competitive with its rivals.

\section{Robot path planning}

The fundamental purpose of robot route planning using optimization criteria is to identify a path from start to finish that is free of collisions [5]. In its most basic form, path planning attempts to build a collision-free path for the robot from its starting place to the destination location while staying away from the strewn about the workspace, using some knowledge about the environment [6]. There are some assumptions made in this work before exploring and proposing a solution to this issue:

- The proposed environment was designed with 200 x 200 pixels.

- The proposed environment was designed with three scenarios free, static \&dynamic obstacles.

- The obstacles are represented by an equally size circular shape.

- The speed of the dynamic obstacles was fixed.

- The mobile robot movement was omnidirectional at any time.

- Minimizing the distance function yields the shortest distance. $f(x, y)[7]$.

$f(x, y)=\sqrt{\left(x_{d}-x_{s}\right)^{2}+\left(y_{d}-y_{s}\right)^{2}}$

where: The next position is represented by $x_{d}, y_{d}$, and the current position is represented by $x_{s}, y_{s}$.

- The shortest path planning was obtained by applying PSO \&ASO algorithms, then compared the results between them. 


\section{Atom Search Optimization}

ASO approach is fundamental molecular dynamics. The notion of molecular dynamics was developed in theoretical physics and has since been applied to domains like chemistry, biology, and materials science; where atomic motion is governed by classical mechanics. Newton's second law is that, if $F_{i}$ is the interaction force and $G_{i}$ is the constraint force acting on the $i^{\text {th }}$ atom and the atom has mass $m_{i}$ then the acceleration of the atom is [8], [4]:

$$
a_{i}=\frac{F_{i}+G_{i}}{m_{i}}
$$

The interaction force operating on the Lennard-Jones potential (L-J potential) is employed on $i^{\text {th }}$ atom from the $j^{\text {th }}$ atom in the $d^{\text {th }}$ dimension at $t$ time, which can be represented as[8], [4]:

$F_{i j}^{d}(t)=\frac{24 \varepsilon(t)}{\sigma(t)}\left[2\left(\frac{\sigma(t)}{r_{i j}(t)}\right)^{13}-\left(\frac{\sigma(t)}{r_{i j}(t)}\right)^{7}\right] \frac{r_{i j}(t)}{r_{i j}^{d}(t)}$

And

$F_{i j}^{\prime}(t)=\frac{24 \varepsilon(t)}{\sigma(t)}\left[2\left(\frac{\sigma(t)}{r_{i j}(t)}\right)^{13}-\left(\frac{\sigma(t)}{r_{i j}(t)}\right)^{7}\right]$

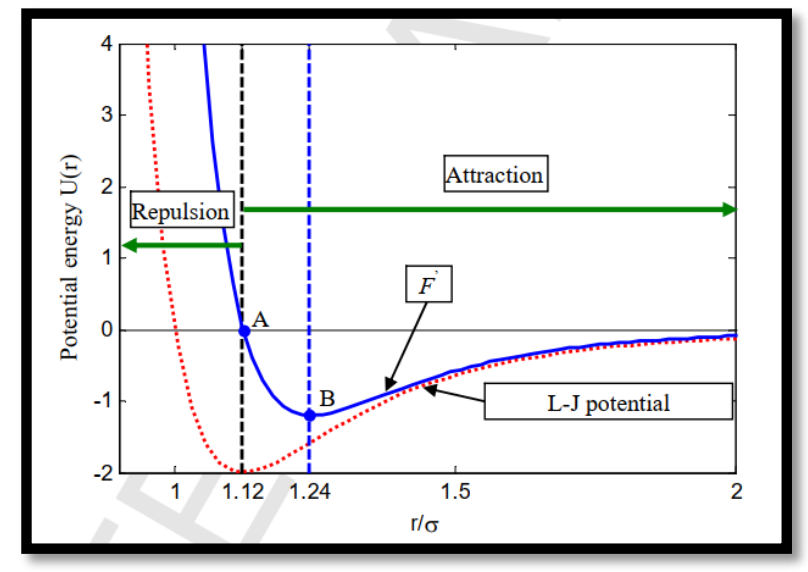

Fig. 1. Atoms force curve

Figure 1 depicts the atoms' force curve for molecular dynamics [4]. It can be demonstrated that due to repulsion or attraction, atoms retain a constant relative distance that fluctuates within a given range, and the repulsion shift relative to the equilibration distance $(\mathrm{r}=1.12 \sigma)$ is substantially larger than the attractiveness. As a result, the repulsion is positive, while the attraction is negative. thus, 
atoms will not converge to a single spot. As a result, equation (4) cannot be directly used to the solution of optimization problems. This equation has been updated to the following as: [8], [4]:

$F_{i j}^{\prime}(t)=-\eta(t)\left[2\left(h_{i j}(t)\right)^{13}-\left(h_{i j}(t)\right)^{7}\right]$

Where the depth function is $\eta(t)$ that can be used to modify the repulsion or attraction zone, defined as [8], [4]:

$\eta(t)=\alpha\left(1-\frac{t-1}{T}\right)^{3} e^{-\frac{20 t}{T}}$

Where the maximum number of iterations is $T$ and $a$ is the depth weight and the function behaviors of $F^{\prime}$ with different values of $\eta$ corresponding to the values of $h$ ranging from 0.9 to 2 are depicted in Figure 2.

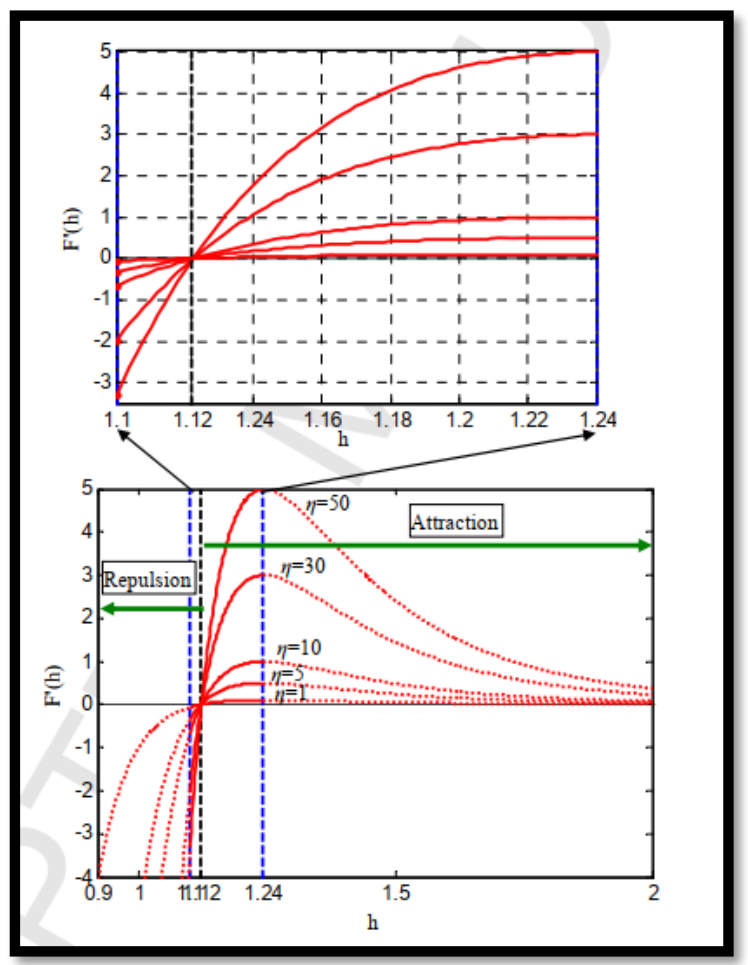

Fig. 2. $F^{\prime}$ function behavior with various values of $\eta$ 
According to Figure 2, repulsion develops when h values are in a certain range of 0.9 to 1.12 , attraction takes place when $\mathrm{h}$ values range from " 1.12 to 2 ". The equilibration takes place when $\mathrm{h}$ values range from 1.12 to 2 . The attraction steadily grows $\mathrm{h}$ from equilibration " $\mathrm{h}=1.12$ " to maximum " $\mathrm{h}=1.24$ " and then starts to reduce. If $\mathrm{h}$ is greater than or equal to 2 , the attraction is about equivalent to zero. As a result, in ASO, to encourage exploration, with a smaller function value, the lower limit of repulsion is set to $h=1.1$, while with a bigger function value, the higher limit of attraction is set to $h=1.2$. With a larger function value, the upper limit of attraction is set at " $h=2.4$ ", therefore $h$ is expressed as [4]:

$h_{\mathrm{ij}}(\mathrm{t})=\left\{\begin{array}{c}\mathrm{h}_{\min } \text { if } \frac{\mathrm{r}_{\mathrm{ij}}(\mathrm{t})}{\sigma(\mathrm{t})}<\mathrm{h}_{\min } \\ \frac{\mathrm{r}_{\mathrm{ij}}(\mathrm{t})}{\sigma(\mathrm{t})} \text { if } \mathrm{h}_{\min } \leq \frac{\mathrm{r}_{\mathrm{ij}}(\mathrm{t})}{\sigma(\mathrm{t})} \leq \mathrm{h}_{\max } \\ \mathrm{h}_{\max } \text { if } \frac{\mathrm{r}_{\mathrm{ij}}(\mathrm{t})}{\sigma(\mathrm{t})}>\mathrm{h}_{\max }\end{array}\right.$

$\mathrm{h}_{\max }$ and $\mathrm{h}_{\min }$ are the upper and lower limits of $\mathrm{h}_{\mathrm{ij}}$ respectively. The length scale $\sigma(\mathrm{t})$ is described as follows [8], [4]:

$\sigma(\mathrm{t})=\left\|\mathrm{x}_{\mathrm{ij}}(\mathrm{t}), \frac{\sum_{\mathrm{j} \in \mathrm{KBest}} \mathrm{x}_{\mathrm{ij}}(\mathrm{t})}{\mathrm{K}(\mathrm{t})}\right\|$

Where: $K_{B e s t}$ denotes the subset of $K$ atoms with the best function fitness values [8], [4].

$\mathrm{h}_{\min }=\mathrm{g}_{0}+\mathrm{g}(\mathrm{t})$

$\left\{\mathrm{h}_{\max }=\mathrm{u}\right.$

where $\mathrm{u}$ is the upper limit and $\mathrm{g}$ is a drift function that aids the algorithm in drifting from exploration to exploitation [8], [4]:

$g(t)=0.1 \times \sin \left(\frac{\pi}{2} \times \frac{t}{T}\right)$

The weighted sum of components of the forces in the $\mathrm{d}^{\text {th }}$ dimension is now the total force acting on the $\mathrm{i}^{\text {th }}$ atom from all other atoms. [8], [4]:

$F_{i}^{d}(t)=\sum_{j \in K B e s t} \operatorname{random}_{j} F_{i j}^{d}(t)$

where random $\mathrm{j}$ is a number in the range $[0,1][8],[4]$.

The geometric restriction plays a crucial role in atom motion in molecular dynamics. For the sake of simplicity, each atom in ASO is assumed to have a covalent link with the best atom, the best atom 
exerts a restriction force on each atom. The constraint force operating on the $\mathrm{i}^{\text {th }}$ atom in the $\mathrm{d}^{\text {th }}$ dimension is defined as [4]:

$G_{i}^{d}=\lambda(t)\left(x_{\text {best }}^{d}(t)-x_{i}^{d}(t)\right)$

where, the Lagrangian multiplier is $\lambda(t)$, which is described as:

$\lambda(\mathrm{t})=\beta \mathrm{e}^{-\frac{20 \mathrm{t}}{\mathrm{T}}}$

Where: the multiplier weight is $\beta$.

As a result, the $\mathrm{i}^{\text {th }}$ acceleration of an atom at time $\mathrm{t}$ can be expressed as [8]:

$$
\begin{gathered}
\mathrm{a}_{\mathrm{i}}^{\mathrm{d}}(\mathrm{t})=\frac{\mathrm{F}_{\mathrm{i}}^{\mathrm{d}}(\mathrm{t})+\mathrm{G}_{\mathrm{i}}^{\mathrm{d}}(\mathrm{t})}{\mathrm{m}_{\mathrm{i}}} \alpha\left(1--\frac{\mathrm{t}-1}{\mathrm{~T}}\right)^{3} \mathrm{e}^{\frac{-20 \mathrm{t}}{\mathrm{T}}} \\
* \sum_{\mathrm{j} \in \text { KBest }} \frac{\operatorname{random}_{\mathrm{j}}\left[2\left(\mathrm{~h}_{\mathrm{ij}}(\mathrm{t})\right)^{13}-\left(\mathrm{h}_{\mathrm{ij}}(\mathrm{t})\right)^{7}\right]}{\mathrm{m}_{\mathrm{i}}(\mathrm{t})} \\
+\beta \mathrm{e}^{-\frac{20 \mathrm{t}}{\mathrm{T}} \frac{\mathrm{x}_{\text {best }}^{\mathrm{d}}(\mathrm{t})-\mathrm{x}_{\mathrm{i}}^{\mathrm{d}}(\mathrm{t})}{\mathrm{m}_{\mathrm{i}}(\mathrm{t})}}
\end{gathered}
$$

At the $\mathrm{t}^{\text {th }}$ repetition, the mass of the $\mathrm{i}^{\text {th }}$ atom is $\mathrm{m}_{\mathrm{i}}(\mathrm{t})$. It is determined as follows [8]::

$$
\begin{aligned}
& M_{i}(t)=e^{-\frac{F i t_{i}(t)-F i t_{\text {best }}(t)}{F i t_{\text {worst }}(t)-F i t_{\text {best }}(t)}} \\
& \mathrm{m}_{\mathrm{i}}(\mathrm{t})=\frac{\mathrm{M}_{\mathrm{i}}(\mathrm{t})}{\sum_{\mathrm{j}=1}^{\mathrm{N}} \mathrm{M}_{\mathrm{j}}(\mathrm{t})}
\end{aligned}
$$

Where: Fit best $(\mathrm{t})=\min _{\mathrm{i}=\{1,2, \ldots, \mathrm{n}\}}$ Fit $_{\mathrm{i}}(\mathrm{t})$ and $\quad$ Fit $_{\text {worst }}(\mathrm{t})=\max _{\mathrm{i}=\{1,2, \ldots, \mathrm{n}\}} \mathrm{Fit}_{\mathrm{i}}(\mathrm{t})$

The $i^{\text {th }}$ atom's location and velocity at time $(\mathrm{t}+1)$ are given by [8]:

$$
\begin{aligned}
& v_{i}^{d}(t+1)=\operatorname{random}_{i}^{d} v_{i}^{d}(t)+a_{i}^{d}(t) \\
& x_{i}^{d}(t+1)=x_{i}^{d}(t)+v_{i}^{d}(t+1)
\end{aligned}
$$

Each atom must communicate with as many atoms as feasible that have a better fitness value than its $\mathrm{K}$ neighbors to improve the exploration in the first stage of the ASO algorithm. The atoms must associate with as few atoms with a higher fitness value as feasible as their $\mathrm{K}$ neighbors to improve exploitation in the last stage of iterations[4]. The distance ratio $\mathrm{r}_{\mathrm{ij}}$ to the length scale $\sigma$ controls 
whether Attraction or repulsion is the attraction or repulsion interaction force acting on each atom from its neighbors. The length scale $\sigma$ reflects the distance between each atom and its K neighbors' average location. As a result, $\mathrm{K}$ is a time-dependent function that decreases with the number of repetitions, and $\mathrm{K}$ may be determined [4], [8]:

$K(t)=N-(N-2) \times \sqrt{\frac{t}{T}}$

\section{Simulation Results}

TO plan the path of a "mobile robot", the proposed ASO algorithm was implemented utilizing MATLAB R2020a. Experiments were carried out On the platform with Intel ${ }^{\circledR}$ Core ${ }^{\mathrm{TM}}$ i7-8550U $\mathrm{CPU}$ at $1.99 \mathrm{GHz}$, Windows $10 \mathrm{Home}$ OS. A number of pre-tests were used to identify the parameters for the suggested procedures as in Table 1. In order to discover the shortest pathways for free, static and dynamic obstacle environments 5 random points were generated. The maximal number of iterations was 20. To resolve the path planning issue, ASO was proposed and compared with a particle swarm optimization algorithm. The study was testing our proposed ASO in three environments (free, Static \& Dynamic obstacles) as in figures3,4,5 \& 6. Search space was $200 \times$ 200 grid with three obstacles. The maximal number of iterations was 20 . The proposed method was run by taking four cases in each environment as in Tables $2,3 \& 4$. From the obtained result, it can be concluded that the execution time(the time required to travel the robot from the current point to the goal) and the minimum path ( minimum cost) in ASO were reduced as compared with PSO algorithms. This demonstrates the Atom Search optimization algorithm's strength.

Table 1. parameters for ASO and PSO

\begin{tabular}{|c|c|c|c|c|c|}
\hline \multirow{2}{*}{$\begin{array}{l}\text { Optimization } \\
\text { algorithm }\end{array}$} & \multicolumn{5}{|c|}{ parameters } \\
\hline & $\begin{array}{c}\text { Maximum } \\
\text { iteration }\end{array}$ & $\operatorname{alpha}(\alpha)$ & $\operatorname{Beta}(\beta)$ & $\operatorname{Limda}(\lambda)$ & $\mathrm{m}$ \\
\hline \multirow[t]{2}{*}{$\mathrm{ASO}$} & 20 & 20 & 0.5 & 1 & 1 \\
\hline & \multicolumn{5}{|c|}{ parameters } \\
\hline \multirow[t]{2}{*}{ PSO } & $\begin{array}{c}\text { Maximum } \\
\text { iteration }\end{array}$ & $\mathrm{W}$ & $\mathrm{C} 1$ & $\mathrm{C} 2$ & Population size \\
\hline & 20 & 1 & 1.5 & 1.5 & 50 \\
\hline
\end{tabular}

\section{5 conclusion}

This work looked at the challenge of mobile robot path planning in a two-dimensional workspace. Atom Search optimization algorithm and Particle Swarm Optimization search methods were proposed for finding the shortest path in the space were employed to select the best path with free 
static and dynamic obstacles. Only the path length was used as objective by using Euclidian distance. The method that has been proposed is ASO was tested in three different settings and shown to be capable of determining the best path with minimum time as compared with PSO. In further research, instead of using ASO, the Butterfly algorithm can be used to optimize the robotic path.

Table 2. comparative between ASO and PSO for free obstacles environment

\begin{tabular}{|c|c|c|c|c|c|c|}
\hline cases & Start point & target & $\begin{array}{c}\text { minimum cost } \\
\text { (Shortest path in } \\
\text { pixels for ASO) }\end{array}$ & $\begin{array}{c}\text { minimum cost } \\
\text { (Shortest path in } \\
\text { pixels for PSO) }\end{array}$ & $\begin{array}{c}\text { Execution time for } \\
\text { ASO } \\
\text { (second) }\end{array}$ & $\begin{array}{c}\text { Execution time for } \\
\text { PSO } \\
\text { (second) }\end{array}$ \\
\hline Case1 & $(20,20)$ & $(180,70)$ & 165 & 173 & 36.0142 & 38.7519 \\
\hline Case2 & $(20,20)$ & $(180,190)$ & 175 & 210 & 41.2211 & 42.7239 \\
\hline Case3 & $(20,10)$ & $(137,95)$ & 122 & 162 & 25.5928 & 32.4814 \\
\hline Case4 & $(20,20)$ & $(30,160)$ & 145 & 146 & 29.9424 & 32.0411 \\
\hline
\end{tabular}

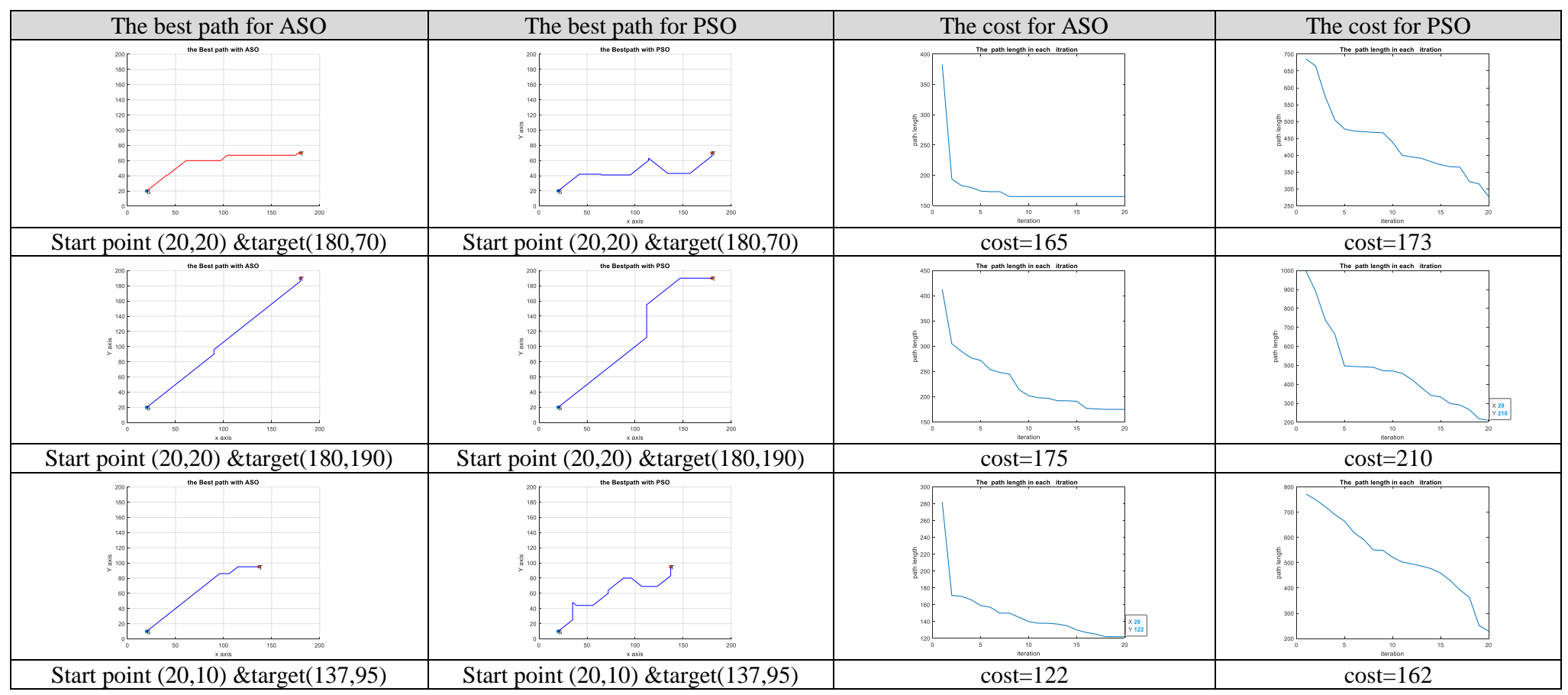




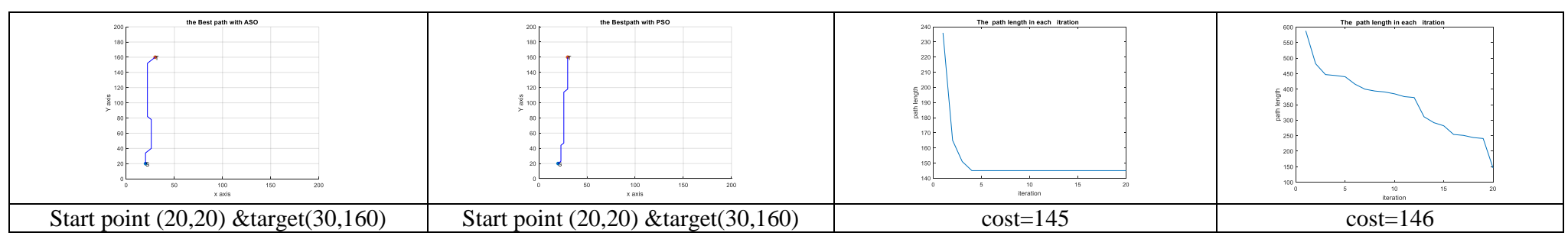

Fig.3. the best path ,the cost in each iteration for ASO \& PSO in free obstacles environment

Table 3. comparative between ASO and PSO for static obstacles environment

\begin{tabular}{|c|c|c|c|c|c|c|}
\hline cases & Start point & target & $\begin{array}{c}\text { minimum cost } \\
\text { (Shortest path in } \\
\text { pixels for ASO) }\end{array}$ & $\begin{array}{c}\text { minimum cost } \\
\text { (Shortest path in } \\
\text { pixels for PSO) }\end{array}$ & $\begin{array}{c}\text { Execution time for } \\
\text { ASO } \\
\text { (second) }\end{array}$ & $\begin{array}{c}\text { Execution time for } \\
\text { PSO } \\
\text { (second) }\end{array}$ \\
\hline Case1 & $(20,20)$ & $(180,70)$ & 166 & 167 & 31.367 & 36.5891 \\
\hline Case2 & $(20,20)$ & $(180,190)$ & 195 & 196 & 37.2071 \\
\hline Case3 & $(20,10)$ & $(137,95)$ & 123 & 139 & 23.5234 & 26.3468 \\
\hline Case4 & $(20,20)$ & $(30,160)$ & 147 & 149 & 27.9884 \\
\hline
\end{tabular}

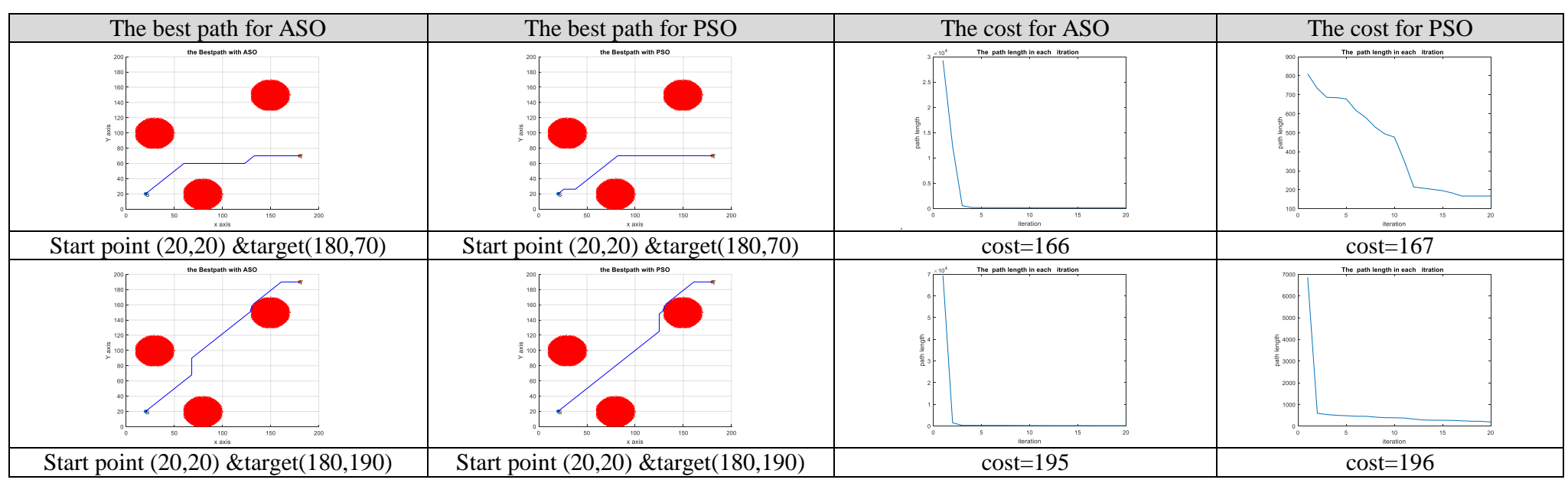




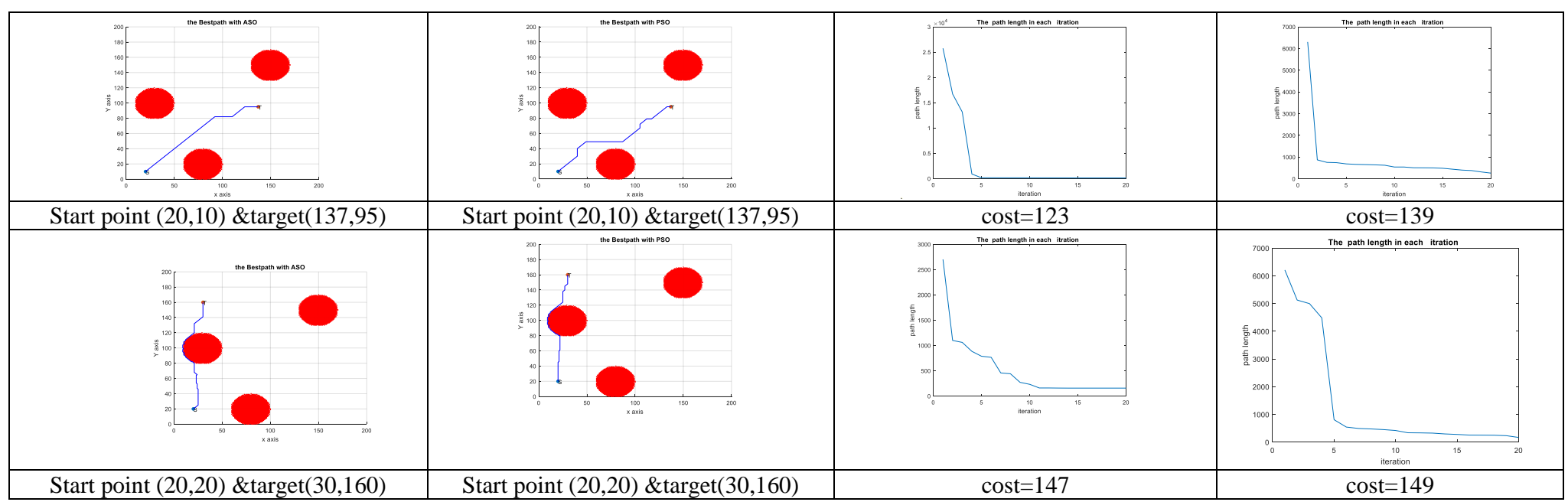

Fig. 4. The best path, the cost in each iteration for ASO \& PSO in static obstacles environment

Table 4. comparative between ASO and PSO for dynamic obstacles environmen

\begin{tabular}{|c|c|c|c|c|c|c|}
\hline cases & Start point & target & $\begin{array}{c}\text { minimum cost } \\
\text { (Shortest path in } \\
\text { pixels for ASO) }\end{array}$ & $\begin{array}{c}\text { minimum cost } \\
\text { (Shortest path in } \\
\text { pixels for PSO) }\end{array}$ & $\begin{array}{c}\text { Execution time for } \\
\text { ASO } \\
\text { (second) }\end{array}$ & $\begin{array}{c}\text { Execution time for } \\
\text { PSO } \\
\text { (second) }\end{array}$ \\
\hline Case1 & $(20,20)$ & $(180,70)$ & 165 & 169 & 24.5218 & 20.2752 \\
\hline Case2 & $(20,20)$ & $(180,190)$ & 195 & 268 & 6.6995 & 22.4291 \\
\hline Case3 & $(20,10)$ & $(137,95)$ & 121 & 161 & 15.7852 \\
\hline Case4 & $(20,20)$ & $(30,160)$ & 145 & 151 & 27.5628 & 5011 \\
\hline
\end{tabular}

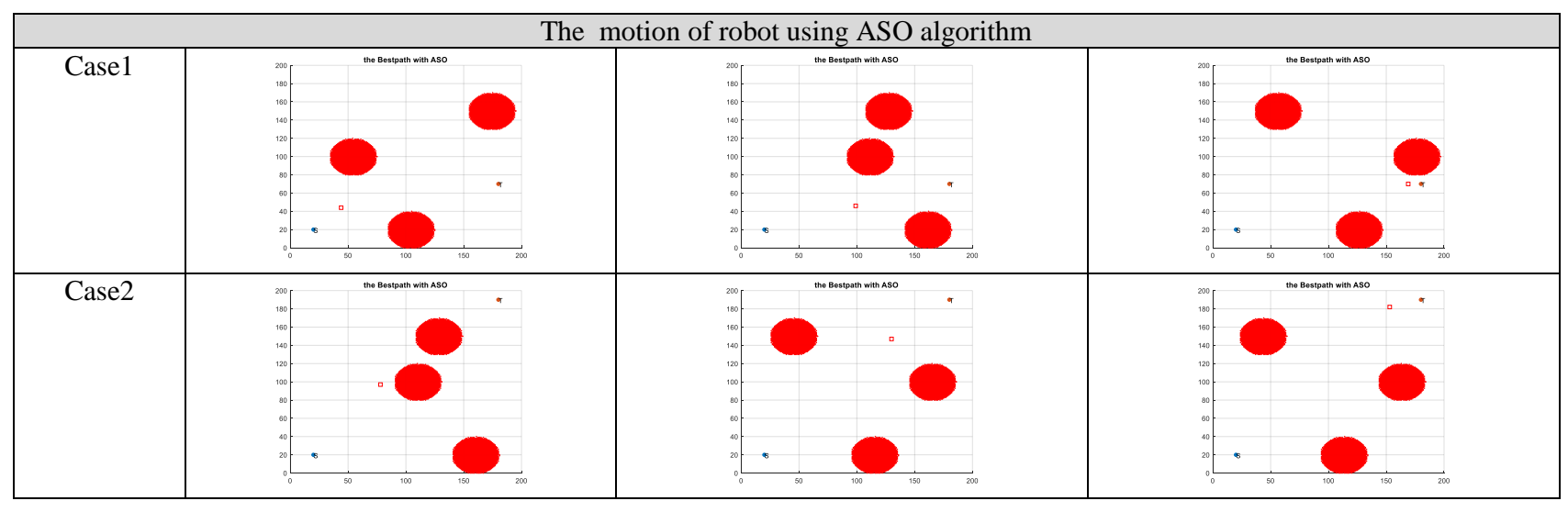




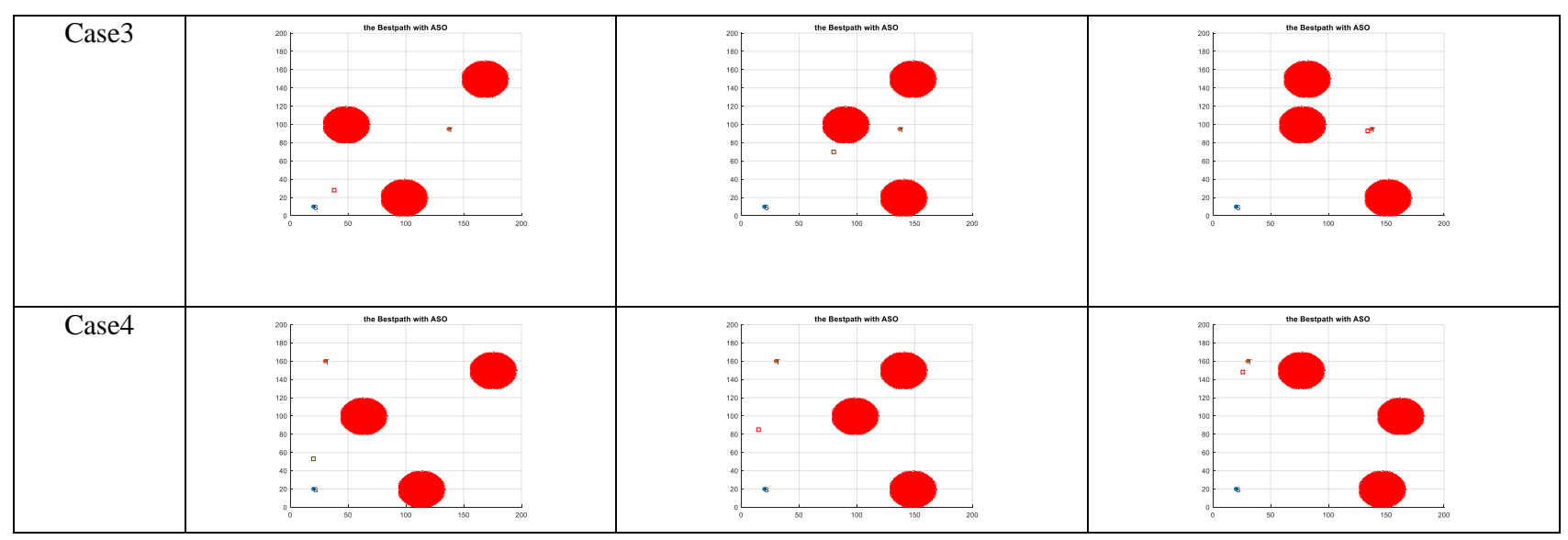

Fig. 5. The robot's motion from start point to end point in each case using ASO

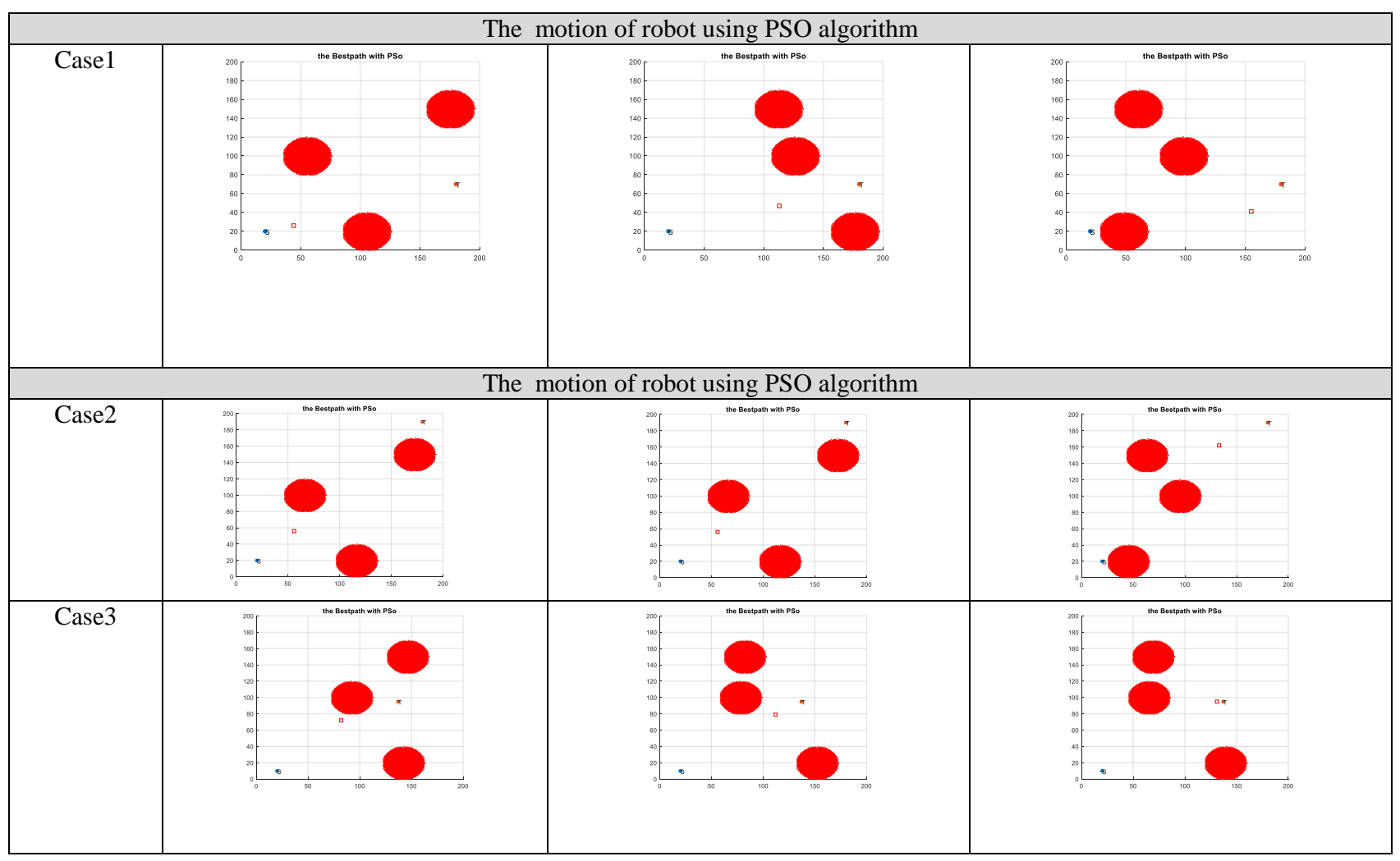




Case4

Fig. 6. The robot's motion from start point to end point in each case using PSO

\section{References:}

[1] Tuba E, Strumberger I, Zivkovic D, Bacanin N, Tuba M. Mobile Robot Path Planning by Improved Brain Storm Optimization Algorithm. 2018 IEEE Congr Evol Comput CEC 2018 - Proc. 2018; (July): $1-8$.

[2] Abed, I. A., Koh, S. P., Sahari, K. S. M., Tiong, S. K., \& Yap, D. F. (2012). Comparison between genetic algorithm and electromagnetism-like algorithm for solving inverse kinematics. World Applied Sciences Journal, 20(7), 946-954.

[3] Yüzgeç U, Doğan L. Robot Path Planning using Gray Wolf Optimizer. 2019; (May 2018).

[4] Zhang H, Lin W, Chen A. SS symmetry Path Planning for the Mobile Robot: A Review. 2018;

[5] Tan, J. D., Dahari, M., Koh, S. P., Koay, Y. Y., \& Abed, I. A. (2016). An improved electromagnetismlike algorithm for numerical optimization. Theoretical Computer Science, 641, 75-84.

[6] Zhao, W., Wang, L., \& Zhang, Z. (2019). A novel atom search optimization for dispersion coefficient estimation in groundwater. Future Generation Computer Systems, 91, 601-610.

[7] Wang Y, Cai F, Wang Y. "Dynamic path planning for mobile robot based on particle swarm optimization Dynamic Path Planning for Mobile Robot based on Particle Swarm Optimization". 2017;020024(August).

[8] Châari I, Koubâa A, Bennaceur H, Trigui S, Al-Shalfan K., SmartPATH: A hybrid ACO-GA algorithm for robot path planning. 2012 IEEE Congr Evol Comput CEC 2012. 2012;(1):10-5.

[9] Ibraheem IK, Ajeil FH, Khan ZH. Path planning of an autonomous mobile robot in a dynamic environment using modified bat swarm optimization. arXiv. 2018; (July).

[9] Ghosh KK, Guha R, Ghosh S, Bera SK, Sarkar R. Atom search optimization with simulated annealingA hybrid metaheuristic approach for feature selection. arXiv. 2020. 